\title{
Envelhecimento Humano e Sensibilidade ao Contraste Fotópica para Frequências Angulares ${ }^{1}$
}

\author{
Thiago Leiros Costa \\ Universidade Federal da Paraíba \\ Renata Maria Toscano Barreto Lyra Nogueira \\ Universidade de São Paulo \\ Anne Gleide Filgueira Pereira \\ Universidade Federal da Paraíba \\ Sandra Helena Ramalho Mousinho \\ Universidade Federal da Paraíba \\ Michelle Madruga Marques \\ UNIPE \\ Natanael Antonio dos Santos 2 \\ Universidade Federal da Paraíba
}

\begin{abstract}
RESUMO - O objetivo deste estudo foi investigar o efeito do envelhecimento na sensibilidade a frequências angulares com luminância fotópica $\left(42,6 \mathrm{~cd} / \mathrm{m}^{2}\right)$. Foram mensuradas curvas de sensibilidade ao contraste em oito adultos jovens (20-29 anos) e oito idosos (60-70 anos) por meio do método psicofísico da escolha forçada. Todos os participantes estavam livres de doenças oculares identificáveis e tinham acuidade visual normal. Os resultados mostraram que o grupo de idosos apresentou alteração significante na faixa de frequências baixas e altas. Concluiu-se que o envelhecimento parece afetar o processamento de frequências angulares baixas e altas em condições de luminância fotópica.
\end{abstract}

Palavras-chave: percepção visual; sensibilidade ao contraste; envelhecimento humano; frequência angular.

\section{Human Aging and Photopic Contrast Sensitivity for Angular Frequencies}

\begin{abstract}
The aim of this study was to investigate the effect of aging on the sensitivity to angular frequencies in photopic luminance $\left(42.6 \mathrm{~cd} / \mathrm{m}^{2}\right)$. Contrast sensitivity curves were measured in eight adults, from 20 to 29 years old and eight elder people, from 60 to 70 years old by means of the forced-choice method. All subjects were free from identifiable ocular disease and had normal visual acuity. The results showed significant decline of the sensitivity of the older adults group to high and low frequencies. It was concluded that aging might affect the processing of high and low angular frequencies in photopic luminance conditions.
\end{abstract}

Keywords: visual perception; contrast sensitivity; human aging; angular frequencies.

Nas últimas décadas, diversas pesquisas vêm reforçando o modelo de canais múltiplos proposto por Campbell e Robson (1968) para tentar explicar como o sistema visual humano (SVH) e de outros mamíferos processa a forma dos objetos (De Valois \& De Valois, 1988). Esse modelo defende que o SVH é composto por uma série de vias ou canais sintonizados seletivamente para bandas estreitas de frequências espaciais. Assim, na percepção de cenas visuais complexas (ou objetos), o SVH decompõe os estímulos em seus componentes mais elementares (ondas senoidais e cossenoidais), em um processo de análise que se inicia na retina. Em seguida, as

1 Trabalho apresentado na XXI Reunião Anual da Federação de Sociedades de Biologia Experimental (FeSBE). Pesquisa financiada pelo CNPq através de Bolsa de Produtividade em Pesquisa (Processo: 304716/2007-3).

2 Endereço para correspondência: Laboratório de Percepção, Neurociências e Comportamento (LPNeC), Depto. de Psicologia, Universidade Federal da Paraíba. Campus I, Cidade Universitária. João Pessoa, PB. CEP 58051-900.E-mail: natanael.santos@pesquisador.cnpq.br. informações são transmitidas por vias especializadas até o córtex, onde ocorre um processo de síntese, interpretação, representação e conhecimento dos objetos. Uma discussão mais abrangente acerca do modelo de canais múltiplos e do uso da análise de sistema linear na percepção visual pode ser encontrada em De Valois e De Valois (1988).

Partindo desse referencial teórico, a função de sensibilidade ao contraste (FSC) pode ser definida como "o envelope de sensibilidade para a série total de canais, cada um sensível a uma faixa restrita e discreta do espectro de frequência espacial” (Santos \& Simas, 2001, p. 589). A FSC é uma ferramenta utilizada para caracterizar a resposta do sistema visual em níveis críticos de contraste, pois permite medir os limiares de detecção de contraste para estímulos elementares de frequências baixas, médias e altas. Nesses termos, a mesma seria um indicador do funcionamento de canais hipotéticos relacionados ao processo de análise e síntese de cenas visuais. Geralmente, em percepção visual de contraste, padrões elementares são formados por uma onda modulada 
senoidal ou cossenoidalmente no espaço e definida pela frequência espacial (variável independente) e pelo contraste (variável dependente). Frequência espacial seria o número de ciclos (listras claras e escuras) por unidade de espaço, geralmente definida em ciclos por grau de ângulo visual (cpg). No caso específico da frequência angular, a mesma é definida pelo número de ciclos por $360^{\circ}$ (Figura 1). Já o contraste pode ser definido na relação entre luminância máxima e mínima (picos e depressões da onda, respectivamente) e ilustrado matematicamente pela equação de Michelson (De Valois \& De Valois, 1988): $\mathrm{C}=\mathrm{L}_{\max }-\mathrm{L}_{\min } / \mathrm{L}_{\max }+\mathrm{L}_{\min }$. As curvas de sensibilidade ao contraste geralmente apresentam um formato bem característico. Elas apresentam um pico na faixa de frequências médias e depressões nas faixas de frequências baixas e altas.

\section{Envelhecimento Humano e FSC}

Diversos estudos descrevem a existência de alterações na FSC ao longo do processo de envelhecimento humano (Higgins, Jaffe, Caruso \& deMonasterio, 1988; Santos, Simas \& Nogueira, 2003; Schefrin, Tregear, Harvey Jr \& Werner, 1999), porém, existem alguns dados discrepantes na literatura, conforme abordado nas revisões de Crassini, Brown e Bowman (1988) e de Owsley, Sekuler e Siemsen (1983). As discrepâncias nos resultados das pesquisas geralmente dizem respeito às faixas de frequências que são mais afetadas pelo processo de envelhecimento, sendo que a variedade de resultados encontrados provavelmente se deve à diversidade de métodos distintos usados na mensuração da FSC. Um exemplo disso pode ser observado no trabalho de Higgins e cols. (1988), que utilizaram dois métodos psicofísicos distintos (métodos dos ajustes e da escolha forçada) para estimar a FSC da mesma amostra. Os dois métodos renderam resultados distintos e, como conclusão, os autores propõem que o método psicofísico do ajuste seja evitado no estudo da FSC.

Independentemente da existência de resultados discrepantes na literatura, algumas hipóteses têm encontrado respaldos: (i) o envelhecimento provoca alterações na sensibilidade às frequências espaciais altas (Fiorentini, Porciatti, Morrone \& Burr, 1996; Schefrin \& cols., 1999); (ii) essas alterações se tornam significantes a partir dos 50 anos de idade (Nio \& cols., 2000; Owsley \& cols., 1983; Santos \& cols., 2003); (iii) as mudanças se devem a alterações não só nos componentes óticos do olho, como também nos componentes neurais envolvidos no processamento visual (Burton, Owsley \& Sloane, 1993; Campbell \& Green, 1965; Habak \& Faubert, 2000; Higgins \& cols., 1988).

\section{O Presente Estudo}

Este estudo se propõe a comparar limiares de contraste entre adultos e idosos, utilizando estímulos de frequências espaciais angulares em condições de luminância fotópica. A maioria absoluta dos estudos que relacionam a FSC ao envelhecimento utiliza estímulos com padrões de grade senoidal (Owsley \& cols., 1983). Os estudos sobre o envelhecimento, utilizando estímulos com configurações radiais e angulares são raros, e, nessas condições de luminância, a utilização de estímulos angulares é inédita. Maiores informações sobre estímulos de frequências angulares, propostos originalmente por Simas (1985), podem ser encontradas na literatura (Santos \& Simas, 2001; Santos \& cols., 2003; Simas \& Dodwell, 1990).

A mensuração da FSC em condições de luminância fotópica proporciona um indicativo da responsividade da via parvocelular, via que recebe aferências principalmente da fóvea e é especializada no processamento dos detalhes finos dos objetos, ou seja, das frequências médias e altas (Benedek, Benedek, Kéri \& Janáky, 2003).

\section{Método}

\section{Participantes}

Participaram deste estudo 16 voluntários: oito adultos jovens de ambos os sexos, com idades entre 20 e 29 anos (M = 23,3; DP = 2,8), designados como Grupo Controle (GC), e oito idosos com idades entre 60 e 70 anos, de ambos os sexos $(\mathrm{M}=65,8 ; \mathrm{DP}=3,27)$, designados como Grupo Experimental (GE). Todos os participantes estavam livres de patologias identificáveis do sistema visual, haviam consultado oftalmologistas nos últimos 12 meses e apresentavam acuidade visual normal ou corrigida, avaliada pela tabela de optotipos "E" de Rasquin no próprio laboratório.

A participação na pesquisa ocorreu mediante assinatura de termo de consentimento livre e esclarecido conforme a Resolução nº 196/96 do Conselho Nacional de Saúde, que trata das diretrizes e normas de pesquisas envolvendo seres humanos. O projeto foi cadastrado na Comissão Nacional de Ética em Pesquisa do Ministério da Saúde (CONEP/MS064608) e aprovado pelo comitê de Ética Local do Centro de Ciências da Saúde.

\section{Equipamentos e estímulos}

Os estímulos foram apresentados em um monitor LG de 19 polegadas, com resolução da tela 1024 x 768 pixels, conectado a um microcomputador Pentium IV por meio de um "hardware" Bits ++ (Cambridge Research Systems) e de uma placa de vídeo com entrada VGA e DVI. A mensuração da luminância média da tela e a correção Gamma do monitor foram feitas utilizando um Fotômetro OptiCAL (Cambridge Research Systems). Além disso, foi utilizada uma cadeira giratória fixada a $150 \mathrm{~cm}$ da tela do monitor. Um programa em linguagem $\mathrm{C}++$ foi desenvolvido no laboratório para gerar os estímulos angulares e controlar os arranjos experimentais.

Foram utilizadas como estímulos de teste as frequências angulares de 3, 24, 48 e 96 ciclos $/ 360^{\circ}$ (ver Figura 1) e como estímulo neutro, um padrão homogêneo com luminância média de 42,6 cd/ $/ \mathrm{m}^{2}$. Todos os estímulos eram circulares, com um diâmetro de 7,25 graus de ângulo visual. 


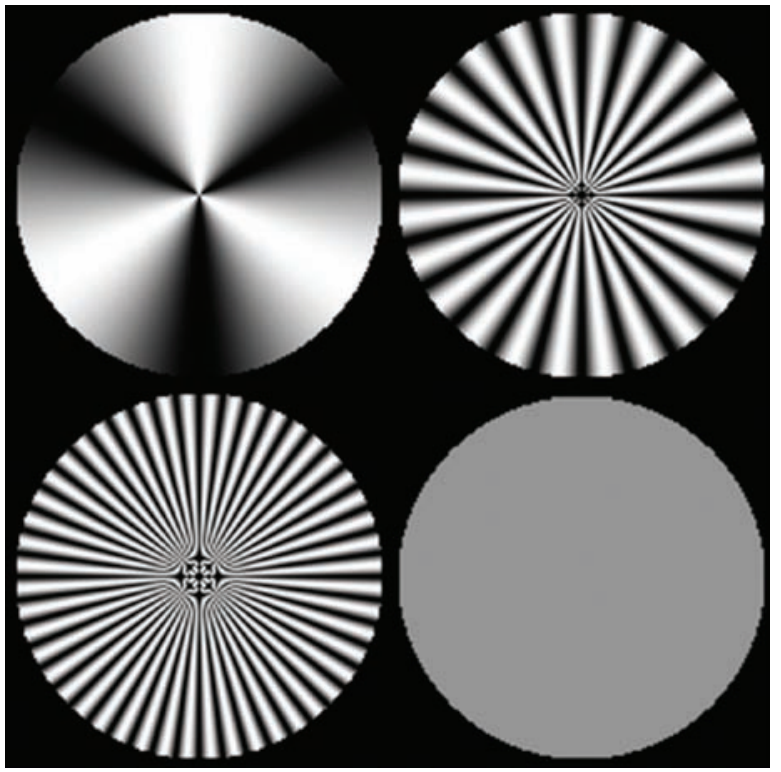

Figura 1. Exemplos de estímulos de frequências angulares. Acima, à esquerda, 3 ciclos $/ 360^{\circ}$ e à direita, $24 \operatorname{ciclos} / 360^{\circ}$. Abaixo, à esquerda, 48 ciclos $/ 360^{\circ}$ e à direita, estímulo homogêneo com a luminância média de $42,6 \mathrm{~cd} / \mathrm{m} 2$. Estímulos originalmente calibrados para serem vistos a 150 cm de distância.

\section{Procedimento}

Utilizou-se um delineamento experimental com medidas repetidas como é peculiar às pesquisas em percepção visual com método psicofísico, onde o "N" estatístico é obtido a partir do número de reversões ou valores de máximos e mínimos de contraste. O delineamento experimental foi composto por duas variáveis independentes (frequências angulares e idades) e uma variável dependente (limiar ou sensibilidade ao contraste).

As medidas foram realizadas com o método psicofísico da escolha forçada (Wetherill \& Levitt, 1965). Esse método se baseia no cálculo da probabilidade de acertos consecutivos por parte do participante, ou seja, em cerca de 100 apresentações de escolhas entre os dois estímulos (estímulo de teste e estímulo neutro), a frequência angular (estímulo de teste) é percebida em aproximadamente $79 \%$ das vezes pelo voluntário. O procedimento para medir o limiar para cada frequência consistiu na apresentação sucessiva simples do par de estímulos, cabendo ao participante escolher dentre eles qual continha a frequência angular. O critério adotado para variar o contraste de cada frequência angular testada foi o de três acertos consecutivos para decrescer uma unidade $(20 \%)$ e um erro para acrescer na mesma unidade.

Durante cada sessão experimental foi apresentada uma sequência de estímulos. Essa sequência era iniciada por um sinal sonoro, acompanhado imediatamente pela apresentação do primeiro estímulo por $2 \mathrm{~s}$; em seguida, havia um intervalo entre estímulos de $1 \mathrm{~s}$, após o qual era apresentado o segundo estímulo por $2 \mathrm{~s}$; finalmente, ocorria a resposta do participante. A ordem de apresentação dos estímulos era aleatória. Os participantes foram instruídos a pressionar o botão do lado esquerdo (sinalizado com o número 1) do mouse quando a frequência angular era apresentada primeiro e o botão do lado direito (sinalizado com o número 2) quando era apresentada em segundo lugar, isto é, após o estímulo neutro. Os sinais sonoros que indicavam a escolha correta e o início da apresentação do par de estímulos eram diferentes. A sessão experimental era concluída automaticamente quando era obtido um total de seis reversões (ou seis valores de contraste, sendo três máximos e três mínimos).

Cada uma das frequências angulares foi estimada pelo menos duas vezes (duas sessões experimentais), em dias diferentes, por cada um dos participantes. Em média, 16 curvas foram medidas para cada grupo de voluntários, totalizando 32 curvas. Todas as estimativas foram medidas à distância de 150 cm, com visão binocular.

\section{Resultados e Discussão}

Após cada sessão, o programa produziu uma folha de resultados com a situação experimental e os seis valores de contraste obtidos. Os valores de contraste obtidos para cada frequência foram agrupados em planilhas por faixa etária e a grande média utilizada como estimativa do limiar de contraste (ou sensibilidade ao contraste) em função de cada frequência angular testada.

A Figura 2 mostra as curvas de sensibilidade ao contraste para os dois grupos. O limiar de contraste pode ser definido como 1/FSC, ou o inverso da curva de sensibilidade ao contraste. Assim, altos valores de limiar são indicativos de baixa sensibilidade e vice-versa. As barras verticais em cada uma das curvas indicam os erros padrões das médias. Observa-se que a sensibilidade máxima ocorreu na faixa de frequência angular de 24 ciclos $/ 360^{\circ}$ para os dois grupos, com reduções na sensibilidade ao contraste nos extremos das curvas.

AANOVA para medidas repetidas indicou um efeito significante de frequência $\left(\mathrm{F}_{(3,570)}=217,57 ; \mathrm{p}<0,001\right)$, de idade $\left(F_{(1,190)}=108,59, \mathrm{p}<0,001\right)$ e uma interação entre idade $\mathrm{e}$ frequência $\left(\mathrm{F}_{(3,570)}=34,68, \mathrm{p}<0,001\right)$. O teste post-hoc Tukey HSD indicou diferenças significantes entre as respostas dos dois grupos nas frequências de 3 e 96 ciclos $/ 360^{\circ}$.

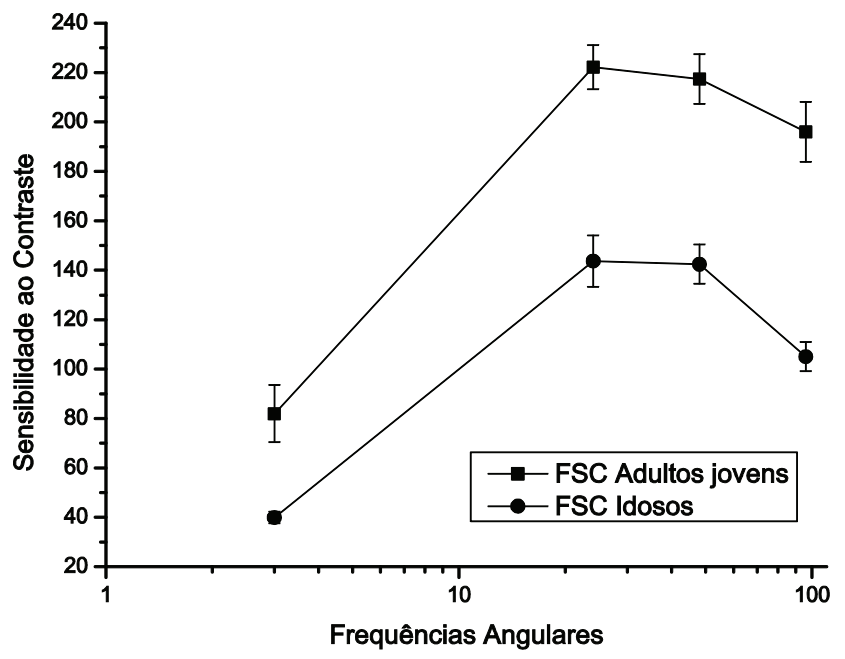

Figura 2. Curvas de sensibilidade ao contraste para frequências angulares em adultos (-—) e idosos (- - - . As linhas verticais mostram o erro padrão da média para cada frequência angular (3, 24, 48 e 96 ciclos $\left./ 360^{\circ}\right)$. 
Esses resultados mostram reduções na sensibilidade do SVH para frequências angulares baixas e altas (3 e 96 ciclos $/ 360^{\circ}$ ) relacionadas ao processo de envelhecimento. Por exemplo, a sensibilidade do GC (adultos) na frequência de 3 ciclos $/ 360^{\circ}$ foi da ordem de 1,8 vez maior que a do GE (idosos), enquanto que a sensibilidade do GC na frequência de 96 ciclos $/ 360^{\circ}$ foi da ordem de 1,5 vez maior que o GE. Assim, observa-se que a maior diferença entre os dois grupos ocorreu na faixa de frequências mais baixas. Por outro lado, os resultados não mostraram alterações significantes entre os dois grupos nas frequências intermediárias (e.g., 24 e 48 ciclos $/ 360^{\circ}$ ), o que indica que a faixa de maior sensibilidade ao contraste é preservada pelo processo de envelhecimento.

Esses resultados com frequências angulares corroboram dados obtidos com estímulos de grade senoidal que também mostram alterações significantes relacionadas à idade, principalmente na faixa de frequências altas. Por outro lado, na faixa de frequências baixas, os resultados são distintos, na medida em que diversos estudos com grades senoidais não relatam alterações significantes nas frequências baixas associadas ao envelhecimento (Owsley \& cols. 1983; Santos, Oliveira, Nogueira, Cruz \& Simas, 2007). Entretanto, os estímulos de frequências angulares e os de frequências espaciais com grade senoidal são diferentes. Enquanto os estímulos angulares são definidos em ciclos por $360^{\circ}$, as frequências espaciais são definidas em ciclos por graus de ângulo visual. Assim, não é possível comparar as curvas de sensibilidade desses dois estímulos visuais, pois as frequências angulares são adimensionais (não dependem da distância, ver Figura 1), enquanto que as frequências espaciais de grade senoidal são dimensionais (dependem da distância). Além disso, estudos psicofísicos e eletrofisiológicos sugerem que esses padrões são processados por áreas visuais distintas, ou seja, grade senoidal seria processada pela área visual V1 enquanto frequência angular seria processada por V4 e pelo córtex ínfero-temporal (Gallant, Connor, Rakshit, Lewis \& Van Essen, 1996; Simas, Santos \& Thiers, 1997).

Os resultados obtidos neste estudo também são distintos daqueles apresentados por Santos, Simas e Nogueira (2004), com método e estímulos angulares semelhantes. Santos e cols. encontraram que a FSC dos idosos foi melhor que a dos adultos jovens na faixa de frequências angulares baixas ( 2 e 4 ciclos $/ 360^{\circ}$ ). Essa disparidade pode se dever às condições diferentes de luminância, pois Santos e cols. utilizaram luminância media de $6,9 \mathrm{~cd} / \mathrm{m}^{2}$ enquanto este estudo utilizou luminância média de $42,6 \mathrm{~cd} / \mathrm{m}^{2}$. Entretanto, embora seja muito cedo para fazer qualquer afirmação, sabe-se que a FSC depende das condições de visualização, pois o processamento visual de objeto e contraste envolve pelo menos dois sistemas: a via visual parvocelular, que é especializada no processamento de frequências espaciais médias e altas ou detalhes finos e opera em níveis altos de luminância, e a via visual magnocelular, que é especializada no processamento de frequências espaciais baixas e opera em níveis baixos ou escotópicos de luminância (Benedek \& cols., 2003; De Valois \& De Valois, 1988). Não se sabe ainda como o envelhecimento interage com níveis de luminância diferentes ou com as vias parvo e magnocelular e novas pesquisas precisam ser realizadas para investigar melhor esse fenômeno.

\section{Referências}

Benedek, G., Benedek, K.. Kéri, S., \& Janáky, M. (2003). The scotopic low-frequency spatial contrast sensitivity develops in children between the ages of 5 and 14 years. Neuroscience Letters, 345, 161-164.

Burton, K. B., Owsley, C., \& Sloane, M. E. (1993). Aging and neural spatial contrast sensitivity: Photopic vision. Vision Research, 33, 936-946.

Campbell, F. W., \& Green, D. G. (1965). Optical and retinal factors affecting visual resolution. Journal of Physiology, 181, 576-593.

Campbell, F. W., \& Robson, F. G. (1968). Application of the Fourier analysis to the visibility of gratings. Journal of Physiology, 197, 551-566.

Crassini, B., Brown, B., \& Bowman, K. (1988). Age-related changes in contrast sensitivity in central and peripheral retina. Perception, 17, 315-332.

De Valois, R. L., \& De Valois, K. K. (1988). Spatial vision. New York: Oxford University Press.

Fiorentini, A., Porciatti, V., Morrone, M. C., \& Burr, D. C. (1996). Visual ageing: Unspecific decline of the responses to luminance and colour. Vision Research, 21, 3557-3566.

Gallant, J. L., Connor,C. E., Rakshit, S., Lewis, J. W., \& Van Essen, D. C. (1996). Neural responses to polar, hyperbolic, and Cartesian gratings in area V4 of the macaque monkey. Journal of Neurophysiology, 76, 2718-2739.

Habak, C., \& Faubert, J. (2000). Larger effect of aging on the perception of higher-order stimuli. Vision Research, 40, 943-950.

Higgins, K. E., Jaffe, M. J., Caruso, R. C., \& deMonasterio, F. M. (1988). Spatial contrast sensitivity: Effects of age, test-restest and psychophysical method. Journal of the Optical Society of America A, 12, 2173-2180.

Nio, Y. K., Jansonius, N. M., Fidler, V., Geraghty, E., Norrby, S., \& Kooijiman, A. C. (2000). Age-related changes of defocusspecific contrast sensitivity in healthy subjects. Ophtalmic and Physiological Optics, 20, 323-334.

Owsley, C., Sekuler, R., \& Siemsen, D. (1983). Contrast sensitivity throughout adulthood. Vision Research, 23, 689-699.

Santos, N. A., Oliveira, A. B., Nogueira, R. M. T. B. L., Cruz, E. D. N., \& Simas, M. L. B. (2007). Efeito da idade na percepção visual de grades senoidais em luminância baixa. Psicologia: Reflexão e Crítica, 19, 415-421.

Santos, N. A., \& Simas M. L. B. (2001). Função de sensibilidade ao contraste: Indicador da percepção visual da forma e da resolução espacial. Psicologia: Reflexão e Crítica, 14, 589-597.

Santos, N. A., Simas, M. L. B., \& Nogueira, R. M. T. B. L. (2003). Processamento visual da forma em idosos: curvas de limiar de contraste para frequências angulares e senoidais. Psicologia: Reflexão e Crítica, 16, 271-277.

Santos, N. A, Simas, M. L. B., \& Nogueira, R. M. T. B. L. (2004). Comparison of angular frequency contrast sensitivity in young and older adults. Brazilian Journal of Medical and Biological Research, 37, 375-378.

Schefrin, B. E., Tregear, S. J., Harvey Jr, L. O., \& Werner, J. S. (1999). Senescent changes in scotopic contrast sensitivity. Vision Research, 22, 3728-3736.

Simas, M. L. B. (1985) Linearity and domain invariance in the visual system. Tese de Doutorado, Queens University, Kingston.

Simas, M. L. B., \& Dodwell, P. C. (1990). Angular frequency filtering: A basis for pattern decomposition. Spatial Vision, 5, 59-74. 
Simas, M. L. B., Santos, N. A., \& Thiers, F. A. (1997). Contrast sensitivity to angular frequency stimuli is higher than that for sinewave gratings in the respective middle range. Brazilian Journals of Medical and Biological Research, 30, 633-636.

Wetherill, G. B., \& Levitt H. (1965). Sequential estimation of points on a psychometric function. The British Journal of Mathematical and Statistical Psychology, 18, 1-10.
Recebido em 09.06.08

Primeira decisão editorial em 09.06.08

Aceito em 11.05.09 